
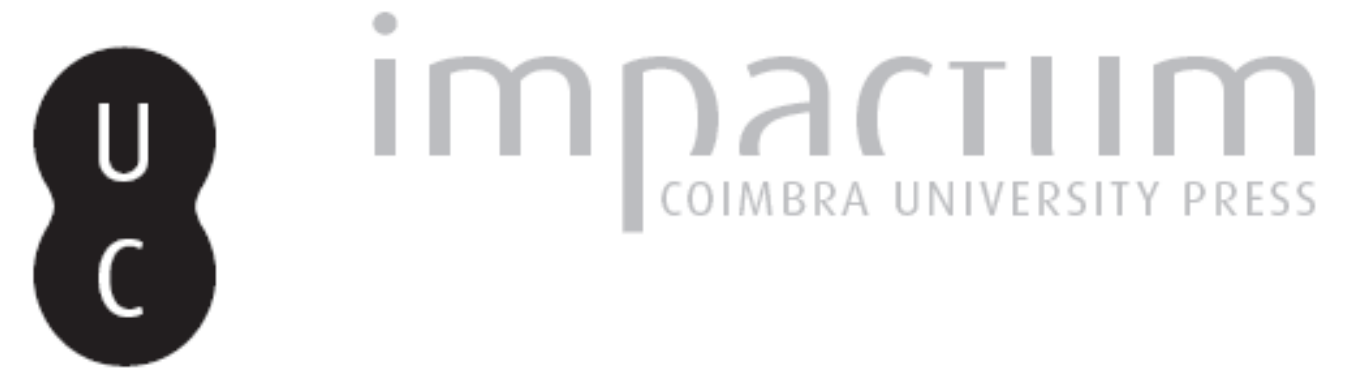

\title{
La danse ou la célébration du monde
}

\section{Autor(es): $\quad$ Breton, David le}

Publicado por: CIAS - Centro de Investigação em Antropologia e Saúde

URL persistente:

URI:http://hdl.handle.net/10316.2/41271

DOI:

DOI:http://dx.doi.org/10.14195/2182-7982_18_1

Accessed : $\quad$ 26-Apr-2023 11:34:36

A navegação consulta e descarregamento dos títulos inseridos nas Bibliotecas Digitais UC Digitalis, UC Pombalina e UC Impactum, pressupõem a aceitação plena e sem reservas dos Termos e Condições de Uso destas Bibliotecas Digitais, disponíveis em https://digitalis.uc.pt/pt-pt/termos.

Conforme exposto nos referidos Termos e Condições de Uso, o descarregamento de títulos de acesso restrito requer uma licença válida de autorização devendo o utilizador aceder ao(s) documento(s) a partir de um endereço de IP da instituição detentora da supramencionada licença.

Ao utilizador é apenas permitido o descarregamento para uso pessoal, pelo que o emprego do(s) título(s) descarregado(s) para outro fim, designadamente comercial, carece de autorização do respetivo autor ou editor da obra.

Na medida em que todas as obras da UC Digitalis se encontram protegidas pelo Código do Direito de Autor e Direitos Conexos e demais legislação aplicável, toda a cópia, parcial ou total, deste documento, nos casos em que é legalmente admitida, deverá conter ou fazer-se acompanhar por este aviso.

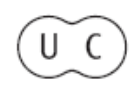


Antropologia Portuguesa

Volume $18 \cdot 2001$

Departamento de Antropologia | Universidade de Coimbra 


\title{
La danse ou la célébration du monde'
}

\author{
David Le Breton \\ Faculté des Sciences Sociales \\ 22 rue René Descartes \\ 67084 Strasbourg cedex, France \\ davidlebreton@evc.net
}

Résumé La danse traditionnelle est une célébration du fait d'exister, elle rend hommage aux dieux, au cosmos, au lien social. Elle s'inscrit dans la mémoire d'une communauté. La danse moderne ne pouvait apparaitre que dans une société de type individualiste, elle est un acte de création qui ne doit qu'à lui même. Elle met en jeu le corps ou le rapport au monde mais sous une forme plus critique, elle est plutôt dans l'interrogation des choses du monde et du social.

Mots-clefs Corps; individu; tradition; création.

Resumo A dança tradicional é uma celebração do facto de existir, ela presta homenagem aos deuses, ao cosmos, ao laço social. Ela inscreve-se na memória de uma comunidade. A dança moderna só poderia aparecer numa sociedade individualista, é um acto de criação que não depende senão de si mesmo. Ela põe em jogo o corpo ou a relação com o mundo mas numa forma mais crítica, ela é sobretudo a interrogação das coisas do mundo e do social.

Palavras-chave Corpos; indivíduo; tradição; criação.

1 Ce texte reprend une confërence faite le 5 avril 2002 lors du forum du GEFAC (Grupo de Etnografia e Folclore da Academia de Coimbra) "Os territórios da dança». 
La danse est célébration du monde, consécration du fait tranquille d'exister et une forme d'offrande au monde et aux autres, un contre-don au fait de vivre. La danse est d'autant plus précieuse qu'elle est inutile, qu'elle ne rapporte rien, qu'elle incarne justement le prix des choses sans prix. Elle nous rappelle que nous sommes homo ludens bien avant d'être homo faber. Des hommes du don, comme le rappelait Mauss (1950) plutôt que des hommes du profit, du rendement, de l'efficacité, de l'urgence. Je ne suis pas danseur, je n'ai pas ce privilège. Mais pourtant la danse m'a souvent accompagné. Avant d'en interroger quelques figures, $j$ 'aimerais faire un bref détour biographique.

Il y a longtemps, un jour de cafard au bord de l'Atlantique, tandis que je regardais la mer du haut d'une dune, j'ai vu une jeune femme s'approcher de la plage et danser à la limite de l'eau et du sable. J'ignore s'il s'agissait de haute technicité ou d'une série de mouvements ordinaires, tout cela est trop loin et je n'ai guère de compétence à en juger. Je sais seulement l'enchantement éprouvé, l'alliance nouée avec le monde et mon émotion de retrouver le frémissement des choses. Elle rappelait la lumière de la mer, la douceur du sable, le contact de l'eau fraiche sur la peau avide. Elle éveillait la sensorialité du monde et le sentiment d'exister. Je suis resté un spectateur ébloui. Cet envol de la matière qui prend la matière comme appui et y retourne en la transfigurant, l'image était belle, j'en ai fait provision, trente ans après elle est toujours vivante. La danse est une noce (parfois tumultueuse) entre un lieu et un corps. Qu'il s'agisse de la scène, d'une ville, d'un champ, d'une forêt, le danseur intègre l'espace dans son corps et se le subordonne comme une matière, un miroir dont il se joue. Il invente l'espace oul il se produit, il le rend visible, et simultanément il est déterminé par lui. La danse est un culte rendu au génie des lieux.

Quelques années plus tard, alors que je pensais encore identifier ma vie au cinéma, j'ai écrit et tourné un moyen métrage en $16 \mathrm{~mm}$ : Seul détruire est au delà des yeux, dans des conditions semi-professionnelles. Je ne parlerai ici du titre (parfaitement cohérent) ni de son contenu, le propos n'est pas là. Mais la figure centrale du film était une jeune danseuse tourangelle: Véronique Gouset, elle y incarnait à la fois le cri et la lumière du monde. Dans l'un des plans, tourné en live, elle marche sur les trottoirs de la ville d'une démarche à la fois gauche et habile avant d'opposer soudain sa danse à la pesanteur et à l'étonnement de la foule. Dans le plan 
final elle court vers la mer de manière à la fois précipitée et déterminée et le film s'achève sur ce contact brutal et chaleureux entre le corps et les vagues tandis que la voix off, la mienne, disait: "Et nous danserons sur la tête des rois". Je revois cette course éperdue et heureuse vers la mer. Dans un autre plan, Véronique devait courir un moment dans les dunes tandis que la caméra, installée sur une voiture la suivait en travelling. Nous avons cherché en vain un lieu propice. Partout où nous pouvions tourner, des genêts tapissaient le sol. Véronique a fait quelques pas pieds nus, mais elle a d'abord renoncé. Il était difficile de tourner ce plan si elle était chaussée. Depuis le début du film elle portait les mêmes vêtements et elle allait pieds nus. Le film était sans concession dans les moyens de traduire le cri: les images, le texte, la musique, le rythme, même parfois la manière de filmer. Véronique Gouset était elle-même sans concession. Elle a enlevé ses chaussures, elle a demandé à l'opératrice de se mettre en place. Et elle s'est lancée. Je la revois encore, dans le remord d'avoir écrit une telle scène, mais nous étions les uns et les autres fascinés par le théâtre de la cruauté d'Artaud, chacun de nous se donnait sans compter. Je vois encore cette course de Véronique, cette manière de mettre des ailes au désespoir et de se retourner sur lui pour le narguer. Comment ne pas l'imaginer danser là où elle est aujourd'hui. Elle est morte à Tours il y a quelques années frappée par la maladie. J'ose espérer que la danse qui l'animait n'a pas cédé devant la douleur et l'imminence du dernier pas. Maintenant elle danse sur la tête des rois.

Au même moment ou peu après, je ne sais plus, je publiais mon premier livre, un roman La Danse Amazonienne (1983) où une danse solitaire face au fleuve est au coeur d'un moment décisif au cours duquel les personnages basculent vers la vie, choisissent désormais d'exister et cessent d'être saisis dans la pesanteur des choses. La danse me touche, mais elle me laisse sans voix, dans une fascination dont je peine à sortir malgré les compétences que l'on me prête généreusement en matière d'anthropologie du corps. Est-ce parce que moi même je ne danse pas que je reste au bord du mystère sans pouvoir me dissoudie en lui. Je confesse que même saisi il y a quelques années dans les foules du carnaval de Récife, tandis que ma compagne dansait et regrettait de me voir sur la berge de la coulée d'effervescence, je regardais avec émotion les enfants et les vieux se perdre dans la jubilation des sens sans pouvoir les rejoindre, les adultes rayonnaient, le corps ruisselant de sueur et de joie. Cette année là, je filmai 
aussi le carnaval avec une vieille caméra $16 \mathrm{~mm}$ mécanique. A cette époque le cinéma était ma manière de danser, j'espère parfois qu'il en reste quelque chose dans l'écriture de mes livres d'anthropologie.

Je suis devant la danse comme Kazantzaki devant la danse de Zorba qu'il aimerait rejoindre sans en avoir les ressources morales. Le vieux Zorba exprime par son corps son amour de la vie. "Pourquoi tu ne ris pas, patron? demanda-t-il. Qu'est-ce que tu as à me regarder? Je suis comme ça. Il y a en moi un diable qui crie et je fais ce qu'il me dit. Chaque fois que je suis sur le point de suffoquer; il crie: "Danse!" et je danse. Et ça me soulage! Une fois, quand mon petit Dimitraki est mort, en Chalcidique, je me suis encore levé comme ça et j'ai dansé. Les parents et les amis qui me regardaient danser devant le corps se sont précipités pour m 'arvêter: "Zorba est devenu fou! qu'ils criaient, Zorba est devenu fou!'" Mais moi, à ce moment là, si je n'avais pas dansé, je serais devem fou de douleur" (Kazantsaki, 1958:105). Je suis sensible à la leçon de Zorba exposant à son compagnon l'universalité de la danse quand elle vise à atteindre l'autre, à lui parler à défaut d'une langue commune. Zorba raconte sa rencontre avec un Russe, ce dernier voulait expliquer la révolution bolchévique, et Zorba ses faits et gestes, son amour de la Crête. Et les deux hommes dansent à tour de rôle: "Ah, mon patvre vieux, ils sont tombés bien bas les hommes. Pouah! Ils ont laissé leurs corps devenir muets, et ils ne parlent plus qu'avec la bouche, mais qu'est ce que tu veux qu'elle dise, la bouche? Si tu avais pu voir; comment il m'écoutait, de la tête aux pieds, le Russe, et comment qu'il comprenait tout! Je lui décrivais, en dansant, mes malheurs, mes voyages, combien de fois je me suis marié, les métiers que j'ai appris: carrier; mineur; colporteur; potier; comitadji, joutur de santouri, marchand de passa-tempo, forgeron et contrebandier; comment on m 'a fouré en prison, comment je me suis évadé, comment je suis arrivé en Russie (...) Tu ris? Tu ne me crois pas, patron? Tut te dis en dedans: dis donc, qu'est ce que c'est que ces boniments qu'il nous débite de Sindbad le marin?' Se parler en dansant, est-ce que c'est possible? Et pourtant, $j$ 'en mettrais ma main au feu, c'est comme ça qu'ils doivent se parler, les dieux et les diables". Un jour, bien sûr je danserai.

J'ai parfois assisté, surtout au Brésil, sur les terreiros d'Umbanda ou de Candomblé à des cérémonies qui m'ont profondément marqué. A mes yeux occidentaux, elles rappellent notre coupure d'individu et la nostalgie inguérissable de la communauté absente dont j'ai longuement parlé dans 
Anthropologie du corps et modernité (Le Breton, 2000). Les danses traditionnelles traduisent la solidarité organique entre soi, l'autre et le cosmos, elles convoquent les dieux, les incarnent, les célèbrent, elles réactualisent les mythes fondateurs, nourrissent la mémoire collective, elles donnent corps à l'innommable et renouvellent l'alliance des hommes et de l'invisible. Elles sanctifient le monde, elles sont comme pour la Grèce antique, un présent des dieux. Elles dessinent dans l'espace et la durée des cérémonies clairement intelligibles d'un épisode à l'autre du rituel, même si des surprises naissent parfois du dialogue avec les dieux ou lors du combat du shaman contre les esprits qu'il affronte. Ce qui se joue dans la danse trouve des interprètes susceptibles d'en expliquer la raison d'être, de traduire la parole des dieux telle qu'elle s'exprime dans un mouvement particulier. La danse traditionnelle est une mise en miroir du cosmos en ce qu'elle célèbre la continuité de toutes ses composantes. Elle est un rite communautaire auquel tous participent par le mouvement ou l'identification. En un mot les danses traditionnelles, dans leur infinie diversité, impliquent une cosmologie (elles disent une vision du monde, inscrivent l'homme au sein de la nature et du cosmos), une anthropologie (elles inscrivent dans l'espace une image de l'homme), une scénographie ou plutôt une ritualité précise; elles sont une création collective et elles renvoient à un temps circulaire, avec des rythmes qui reviennent inlassablement et conduisent à un monde apaisé. Les danses traditionnelles incarnent le monde du "nous-autres", de la communauté, du lien social. Elles se mêlent au temps de la vie collective et de ses rythmes.

La danse contemporaine ${ }^{2}$ est en revanche profondément inscrite dans la question de l'individu, et donc de celle du corps, elle devait attendre pour se déployer avec la force que nous connaissons, l'avènement d'un individualisme croissant dans nos sociétés. Elle est en effet mise en œuvre du corps, pensée en mouvement, écriture singulière de l'espace, jeu de signes, elle n'est donc pas vérité du corps, ou répétition d'un modèle, spontanéité ou énergie en liberté. Elle est même refus de la tradition et

: Certes, les passerelles entre ces deux types de danse sont nombreuses: les formes traditionnelles ne sont jamais immuablement figées dans le temps, elles ne sont pas des musées, elles s'actualisent en maintenant un canevas. Les jeunes générations y apportent leur contribution. La danse contemporaine renouvelle souvent ses figures en puisant dans le répertoire traditionnel, en s'y inspirant. 
recherche inlassablement reprise autour des possibles recelés par le corps. Elle prend acte de la solitude de l'homme dans le monde, c'est-à-dire de l'individualisme; de la fragmentation du monde, de l'histoire qui emporte les sociétés humaines. Pour Laurence Louppe (1997:44), "Il n'y a qu'une seule et vraie danse: celle de chacun... Le danseur moderne et contemporain ne doit sa théorie, sa pensée, son élan, qu'à ses propres forces". Et elle cite Carolyn Brown, partenaire de Merce Cunningham, pour qui "la seule vraie tradition, c'est de tout recommencer à partir de ses propres ressources". Là où la danse traditionnelle est émanation d'une création collective, les créations contemporaines sont signées. La danse en ce sens est infinie car elle incarne toujours provisoirement un segment de l'infinité possible des ressources corporelles. Le chorégraphe ou le danseur avance sur le fil du rasoir, il délaisse les codes sociaux qui protègent de l'angoisse et rendent le monde familier et hospitalier à l'homme. Entre ses mains, il tient cependant un balancier, son style, c'est-à-dire le chemin propre qu'il trace dans la zone à demie jalonnée de son exercice (histoire de la danse, données techniques et scénographiques de son temps, groupe d'influence auquel il se rattache, marché, etc.). Le franchissement constant de cette ligne d'ombre que la danse impose ne se fait pas sans boussole, mais il est vrai que les boussoles ne suffisent pas à elles seules à éloigner les tempêtes.

La danse contemporaine construit d'oeuvre en oeuvre un savoir en marche, une boîte à outils qui permet une lecture des spectacles, une analyse de leur apport, de leur fidélité à un style d'auteur, de leur rupture, de leur métissage, ou de leur conservatisme. Mais la danse contemporaine, contrairement au théâtre, manifeste une symbolique éloignée en principe des codes culturels qui alimentent la vie quotidienne, elle met en oeuvre un corps libéré de la symbolique corporelle qui fonde les échanges de sens entre les individus dans la vie courante. C'est pourquoi elle touche, fascine, émerveille ou inquiète. Le privilège de la danse est de donner à voir à travers les interstices du réel, d'inventer des corps inédits, surprenants ou en relation de miroir déformant. La danse prend le relais de la parole, de la pensée, là où celles-ci restent sans voix, mais loin de désarmer ce silence, elle l'étend. Le monde naît alors à des significations autres, son évidence première se dissout. Le corps apparaît plus que le corps, le monde plus que le monde. La déliaison du symbolisme social restitue le corps aux remous, aux ambivalences, au pulsionnel que les 
codes sociaux visent justement à conjurer. La danse se donne parfois comme morsure ou caresse, elle nous "touche", de toutes manières, mais le sens de la création n'est pas de pacifier les conflits ou les abîmes qui s'ouvrent en chaque homme, il est de "fixer des vertiges", de creuser dans la trame serrée de nos gardes fous des zones de turbulences qui nous mettent autrement au monde et posent à l'homme la question de sa propre question à travers son corps même, c'est-à-dire la condition de son être au monde. Quels corps viennent au monde lorsque le texte social est gommé et que le danseur pousse son exploration en surmontant ses craintes? Chaque création nous offre un chiffre de ce territoire de l'ombre qui commence sous la peau.

Ce que nous enseigne la danse contemporaine c'est l'irréductibilité du corps aux modèles empreints de positivité qui règnent dans la modernité. Le corps est la chair de notre monde. L'homme est son corps et il est plus que son corps, mais ce supplément n'est pas le fait de l'âme ou de l'esprit, il est le fait de l'existence même. La condition humaine est corporelle et notre corps nous demeure un mystère. La danse nous montre un corps bien loin du modèle cartésien qui, sur la base d'une comparaison avec le cadavre, le définit par la surface et l'occupation d'un volume, loin aussi du corps-machine plus ou moins bien relié à une âme rétive, loin aussi de l'anatomo-physiologie qui pèse sur nos représentations occidentales comme le couperet qui distingue radicalement l'homme et son corps. Comme si l'homme n'était que le reste impalpable d'un appendice de chair (Le Breton, 2000). Le questionnement du danseur est anthropologique, le statut du réel est son objet, la question de l'homme et de sa chair son inlassable tâche.

Le corps du danseur n'est pas astreint à la communication, il est affranchi des contraintes de l'identité, même de celles du genre. Il n'est plus assujetti à un statut social, à une filiation, il se construit lui-même dans l'éphémère du geste à travers un jeu de signes. La danse contemporaine est induction d'un sujet en suspens, créant l'espace et le temps où elle se produit, elle est invention de formes et de contenus, matrice éternellement renouvelée du sens plutôt que répétition du même. Elle invente de nouveaux langages ou de nouvelles manières d'être, elle est une exploration sans fin du continent corporel.

Certes, elle n'en est pas moins une construction mentale qui se joue à travers le corps, une intelligence physique du corps, à la manière d'une 
oeuvre écrite dans la série cohérente des mouvements. Avant l'aisance du geste et la transparence du mouvement, il y a l'apprentissage, l'enseignement d'un maître et l'appropriation des techniques corporelles par l'élève. Il y a bien une construction de la grâce ou de la gaucherie (si elle est voulue). L'évidence est acquise: en amont elle est le fait de l'intériorisation des manières élémentaires de se jouer de l'espace, en aval elle réside dans le talent, la capacité d'invention.

De même que les arts plastiques (et notamment le body art) ou le théâtre, la danse participe avec force à l'interrogation lancinante de nos sociétés sur le statut du corps et donc, au delà, sur le statut du sujet dans un monde où il est menacé de toute part. Le danseur coult vers la mer et se tient à la lisière entre le sable et la mer. On se souvient de la dernière phrase de Michel Foucault (1996:398) dans Les Mots et les choses. "L'homme est une invention dont l'archéologie de notre pensée montre aisément la date récente. Et peut-être la fin prochaine. Si ces dispositions venaient à disparaître comme elles sont apparues, si par quelque événement dont nous pouvons tout au plus pressentir la possibilité, mais dont nous ne connaissons pour l'instant encore ni la forme ni la promesse, elles basculaient, comme le fit au tournant du XVIIle siècle le sol de la pensée classique, -alors on peut bien parier que l'homme s'effacerait, comme à la limite de la mer un visage de sable". Mais justement, les pas du danseur imposent d'autres traces ailleurs, se jouent de l'oubli ou de la mort. À la lisière de la mer et du sable, il y a toujours un danseur pour renouveler les pas de l'homme et sauvegarder son visage. La danse est une image d'éternité de l'homme, l'une des formes fondatrices de résistance à la fois critique et jubilatoire de la crise de sens et de valeur de nos sociétés. Même quand elle interroge durement la condition corporelle de l'homme, elle le fait toujours par les moyens du corps. Elle est à l'opposé de ce courant de mépris du corps qui touche en profondeur nos sociétés. Le cogito contemporain de ceux qui n'acceptent pas les impératifs de "l'horreur économique" et de la toute puissance de la technique quand elle rend le monde de moins en moins hospitalier: je danse donc j'existe. Je danse donc mon corps est toujours dans la jouissance du monde (Le Breton, 1999). 


\section{Remerciements}

Je remercie profondément à ce propos João Maria André pour sa confiance.

\section{Bibliographie}

Foucault, M. 1966. Les mots et les choses. Paris, Gallimard.

Kazantsaki, N. 1958. Alexis Zorba. Paris, Livre de poche.

Le Breton, D. 1983. La Danse Amazonieme. Paris, Syros.

Le Breton, D. 1999. L'Adieu au corps. Paris, Métailié.

Le Breton, D. 2000. Anthropologie du corps et modernité. Paris, Presses Universitaire de France.

Louppe, L. 1997. Poétique de la danse contemporaine. Paris, Contredanse.

Mauss, M. 1950. Les techniques du corps, in Sociologie et Anthropologie. Paris, Presses Universitaires de France. 